\title{
IMPROVED PREAMBLE-AIDED TIMING ESTIMATION FOR OFDM SYSTEMS
}

\author{
Adegbenga B. Awoseyila, Christos Kasparis and Barry G. Evans \\ Centre for Communication Systems Research (CCSR), University of Surrey, \\ Guildford GU2 7XH, U.K. \\ a.awoseyila@surrey.ac.uk,c.kasparis@surrey.ac.uk,b.evans@surrey.ac.uk \\ Index Terms: OFDM, preamble, timing synchronization, correlation
}

\begin{abstract}
An improved method for estimating the frame/symbol timing offset in preamble-aided OFDM systems is presented. It uses a conventional preamble structure and combines autocorrelation techniques with restricted crosscorrelation to achieve a near-ideal timing performance without significant increase in complexity. Computer simulations show that the method is robust in both AWGN and fading multipath channels, achieving better performance than the existing methods.
\end{abstract}

\section{Introduction}

Orthogonal Frequency-Division Multiplexing (OFDM) is a popular scheme for fixed and wireless broadband applications due to its robustness against frequency-selectivity in the channel [1]. However, OFDM systems are very sensitive to carrier frequency errors and quite sensitive to timing errors [1]. Consequently, many techniques have been proposed in the past for estimation of these parameters. In this letter, we focus on preamble-aided methods since they give a more robust estimation performance in both continuous and burst applications [2]. 
Schmidl [2] proposed using the autocorrelation of a training symbol with two identical parts to estimate timing and fractional frequency offset. An additional training symbol is then used along with the first to determine the integer frequency offset. However, Schmidl's timing metric has an uncertainty plateau and the method can yield timing estimates which are well beyond the ISI-free region, thus leading to degradation in BER performance [1]. Other autocorrelation methods have been proposed by Park [3], Minn [4] and Shi [5] using different uniquely-designed preamble patterns to obtain sharper timing metrics and improve the timing accuracy. However, they are not robust in fading and strong-ISI channels as will be shown in our simulation results. Therefore, a new method is presented in order to achieve near-ideal accuracy (i.e. timing MSE approaching zero) without significant increase in complexity.

\section{OFDM Signal Description}

The baseband signal samples at the OFDM transmitter output are given by:

$$
x_{k}=\frac{1}{\sqrt{N}} \sum_{n=0}^{N_{\text {use }}-1} X_{n} e^{j 2 \pi k n / N} \quad k=0,1,2,3, \ldots . ., N-1
$$

where $N$ is the total number of subcarriers of which $N_{\text {use }}$ are used, $X_{n}$ represents the data modulated on the $n^{\text {th }}$ subcarrier while $x_{k}$ represents the symbol samples after IFFT processing. Each transmitted OFDM symbol is usually preceeded by a guard interval or cyclic prefix of length $G$ in order to eliminate intersymbol interference (ISI) arising from the wideband channel. 
Assuming sampling precision, the complex-valued samples of the received signal can be represented as:

$r(k)=\sum_{m=0}^{L-1} h(m) x(k-\varepsilon-m) e^{j 2 \pi \Delta f k / N}+w(k)$

where $\varepsilon$ is the timing offset in samples, $\Delta f$ is carrier frequency offset normalized to the subcarrier spacing, $w(k)$ represents the zero-mean complex addditive white Gaussian noise (AWGN) and $h(m)$ is the impulse response of the frequency-selective (ISI) channel whose memory order is $L-1$.

\section{Proposed method}

The proposed method is multi-stage, wherein a simple autocorrelation technique and a novel restricted cross-correlation technique are combined to achieve high estimation performance. A preamble with two identical parts in time-domain (e.g. Schmidl's training symbol $S_{\text {Sch }}$ [2]) is chosen since it provides for low-complexity coarse timing, reliable ML fractional frequency estimation [2] and a wide range integer frequency estimation [6] which is not achievable by the preamble structures of [3-5]. Also, this structure conforms to the WiFi and WiMAX standards wherein only the 2 long identical patterns are used for restricted cross-correlation while the preceding short patterns are excluded via the autocorrelation stage which can differentiate between the short and long patterns.

$$
\begin{aligned}
& S_{S c h}=\left[A_{N / 2} A_{N / 2}\right] \\
& P_{S c h}(d)=\sum_{k=0}^{N / 2-1} r^{*}(d+k) r(d+k+N / 2)
\end{aligned}
$$




$$
\begin{aligned}
& M_{c}(d)=\frac{1}{G+1} \sum_{k=0}^{G}\left|P_{S c h}(d-k)\right|^{2} \\
& \hat{d}_{c}=\underset{d}{\arg \max }\left\{M_{c}(d)\right\}
\end{aligned}
$$

where $A_{N / 2}$ is a random sequence of length $N / 2$ and $P_{S c h}$ is Schmidl's autocorrelation which is integrated in (5) over the length of the cyclic prefix in order to eliminate its uncertainty plateau and achieve a coarse timing metric $M_{c}$ whose peak indicates the coarse timing estimate $\hat{d}_{c}$.

$\hat{d}_{c}$ is subsequently used for frequency offset estimation (pre-FFT and postFFT) via existing techniques such as Schmidl's or Kim's method which are known to give reliable frequency estimates even with a coarse timing $[2,6]$. The total frequency offset is then corrected in the stored received samples, after which cross-correlation is performed. In an ISI channel, coherent crosscorrelation between a frequency-corrected received preamble and its purely random transmitted version will yield a set of very sharp peaks (impulses) which indicate all the arriving paths from the channel $[7,8]$. For a preamble having two identical parts, the coherent cross-correlation will yield three distinct sets of impulses.

Fig. 1 shows an example cross-correlation of the preamble $S_{S c h}$ under no channel distortion, wherein there is a major peak in the middle corresponding to a full-symbol pattern match and two minor peaks at half a symbol away corresponding to a half-symbol pattern match. The minor peaks which are due to the symmetric structure in (4) constitute a major hindrance to using such preamble for cross-correlation timing in a fading and/or noisy channel. This problem is solved using a novel approach as follows: 
The minor cross-correlation peaks occur at a half-symbol timing error and the autocorrelation metric is significantly low at such region [2] as illustrated in Fig. 1. Therefore, the autocorrelation can be used to filter the unwanted minor peaks. One way to achieve this is to simply multiply both metrics together over a restricted timing window of one symbol length, symmetric around the coarse timing estimate $\hat{d}_{c}$ as shown in (7)-(9). This helps to determine the major peak which corresponds to the strongest arriving path. A restricted timing adjustment window is also proposed for the chosen preamble in ISI channels, based on the location of the minor and major cross-correlation peaks and the expected channel delay spread, in order to track the crosscorrelation peak that corresponds to the first arriving channel path with high efficiency as shown in (10)-(11).

The restricted cross-correlation stage is summarized as follows:

$$
\begin{array}{ll}
P_{x}(d)=\sum_{k=0}^{N-1} r_{\text {corT }}(d+k) S_{\text {sch }}^{*}(k) ; & d \in\left\{\hat{d}_{c}-N / 2, \hat{d}_{c}+N / 2\right\} \\
M_{\text {opt }}(d)=\left|P_{x}(d)\right|^{2} \cdot M_{c}(d) ; & d \in\left\{\hat{d}_{c}-N / 2, \hat{d}_{c}+N / 2\right\} \\
\hat{d}_{\text {opt }}=\underset{d}{\arg \max }\left\{M_{\text {opt }}(d)\right\} ; & d \in\left\{\hat{d}_{c}-N / 2, \hat{d}_{c}+N / 2\right\} \\
\hat{d}_{F F T}=\underset{d}{\arg \operatorname{first}}\left\{\left|P_{x}(d)\right|>T_{\text {th }}\right\} ; & d \in\left\{\hat{d}_{\text {opt }}-\lambda, \hat{d}_{\text {opt }}\right\} \\
T_{\text {th }}=\alpha \cdot\left(\operatorname{mean}\left\{\left|P_{x}(d)\right|\right\}\right) ; & d \in\left\{\hat{d}_{\text {opt }}-N / 2+\lambda+1, \hat{d}_{\text {opt }}-\lambda-1\right\}
\end{array}
$$

where $r_{\text {corT }}$ is the total frequency corrected signal and $d$ is chosen in (7-9) to ensure that all relevant timing points that could be the ideal timing are tracked. $d$ is chosen in (10) such as to track the first arriving path $\left(\hat{d}_{F F T}\right)$ which may not 
always be the strongest arriving path $\left(\hat{d}_{\text {opt }}\right)$, wherein all channel paths are expected to be received within $\lambda+1$ samples: $(L-1 \leq \lambda \leq G)$. In (11), $d$ is chosen such as to exclude major and minor peaks and their multipath, in order to calculate a threshold $T_{\text {th }}$ using the mean correlation of neutral timing points. The first arriving channel path (or any other one) is expected to have a correlation which is greater than this threshold. The approach is inherited from [7] based on the statistics of the cross-correlation with $P_{F A}$ being the probability of false alarm and $\alpha=\sqrt{-(4 / \pi) \ln \left(P_{F A}\right)}$. Deriving from extensive simulations, $P_{F A}$ is chosen as $10^{-6}$ similar to [7]. The threshold $T_{\text {th }}$ is used to detect the ideal timing $\hat{d}_{F F T}$.

\section{Computer Simulations}

Computer simulations were performed to verify the performance of the proposed method in comparison to the existing methods. QPSK sub-carrier modulation is used with a normalized frequency offset $\Delta f=0.1$. In Fig. 2, an FFT size: $N=64$, cyclic prefix length: $G=16$ and ISI parameter: $\lambda=0$ is used in an AWGN channel, whereas in Fig. 3, a WiMAX test case of $N=256, G=16$ and $\lambda=16$ is used in an ISI channel consisting of $L=8$ paths with path delays of $\tau_{i}=0,1,2 \ldots L-1$ samples and an exponential power delay profile having average power of $e^{\left(-\tau_{i} / L\right)}$ as used in [1] wherein each path undergoes independent Rayleigh fading. A training symbol with sixteen segments as specified in [4] is used with Minn's method.

Fig. 2 and Fig. 3 show that the proposed method maintains a superior performance to the existing methods in both AWGN and Rayleigh fading ISI 
channels. Although Minn and Shi use a significant increase in computations to achieve an improvement over Schmidl's method, their timing estimates still tend to be biased towards the strongest arriving path and extrinsic timing adjustment is consequently required.

\section{Conclusion}

In this letter, we have presented an improved method for estimating the frame/symbol timing offset in preamble-aided OFDM systems. Results show that its performance is better than the existing methods in both AWGN and fading ISI channels. Furthermore, its preamble structure is compatible with the commercial wireless networking standards.

\section{Acknowledgement}

This work was supported in part by the European SatNEx II programme.

\section{References}

[1] M. Morelli, C.-C.J. Kuo and M.-O. Pun, "Synchronization Techniques for Orthogonal Frequency Division Multiple Access (OFDMA): A Tutorial Review," Proc. of the IEEE, vol. 95, no. 7, pp. 1394-1427, Jul. 2007.

[2] T. Schmidl and D.C. Cox, "Robust frequency and timing synchronization for OFDM," IEEE Trans. Commun., vol. 45, no. 12, pp. 1613 - 1621, Dec. 1997.

[3] B. Park, H. Cheon, C. Kang, and D. Hong, "A novel timing estimation method for OFDM systems," IEEE Commun. Lett., vol. 7, no. 5, pp. 239241, May 2003. 
[4] H. Minn, V. K. Bhargava and K. B. Letaief, "A robust timing and frequency synchronization for OFDM systems," IEEE Trans. W. Commun., vol. 2, no. 4, pp. 822-839, Jul. 2003.

[5] K. Shi and E. Serpedin, "Coarse frame and carrier synchronization of OFDM systems: A new metric and comparison," IEEE Trans. W. Commun., vol. 3, no. 4, pp. 1271-1284, Jul. 2004.

[6] Y. H. Kim, I. Song, S. Yoon and S. Park, "An efficient frequency offset estimator for OFDM systems and its performance characteristics," IEEE Trans. Vehicular Tech., vol. 50, no. 5, pp. 1307-1312, Sep. 2001.

[7] C. Kasparis and B.G. Evans, "A cross-correlation approach for improved timing estimation in OFDM broadcasting systems," 24th AIAA Intl. Commun. Satell. Sys. Conf., ICSSC, pp. 1039-1048, 2006.

[8] A. Fort et al "A performance and complexity comparison of autocorrelation and cross-correlation for OFDM burst synchronization," IEEE Intl. Conf. Acoustics Speech Signal Process, vol. 2, pp. 341-344. 


\section{Figure/Table captions}

Fig. 1: Autocorrelation and cross-correlation metrics for the proposed preamble under no noise and no channel distortion, $N=256, G=16$.

Fig. 2: Timing mean-square-error (MSE) of the estimators in an AWGN channel, $N=64, G=16$.

Fig. 3: Timing mean-square-error (MSE) of the estimators in a Rayleigh fading ISI channel, $N=256, G=16$. 
Figure 1

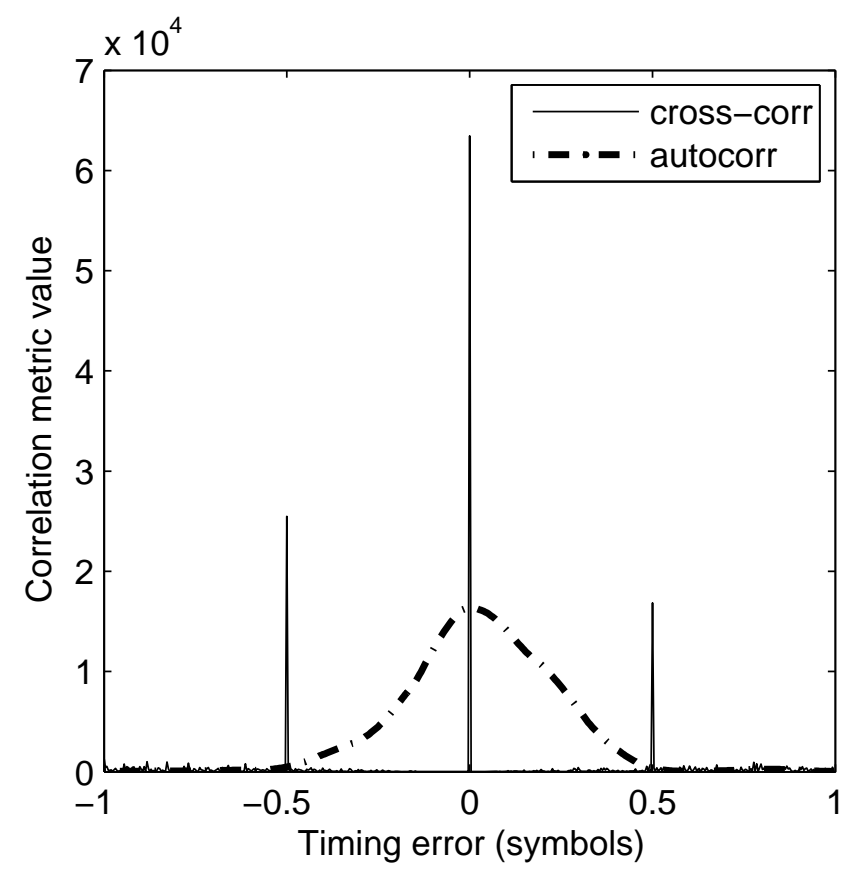


Figure 2

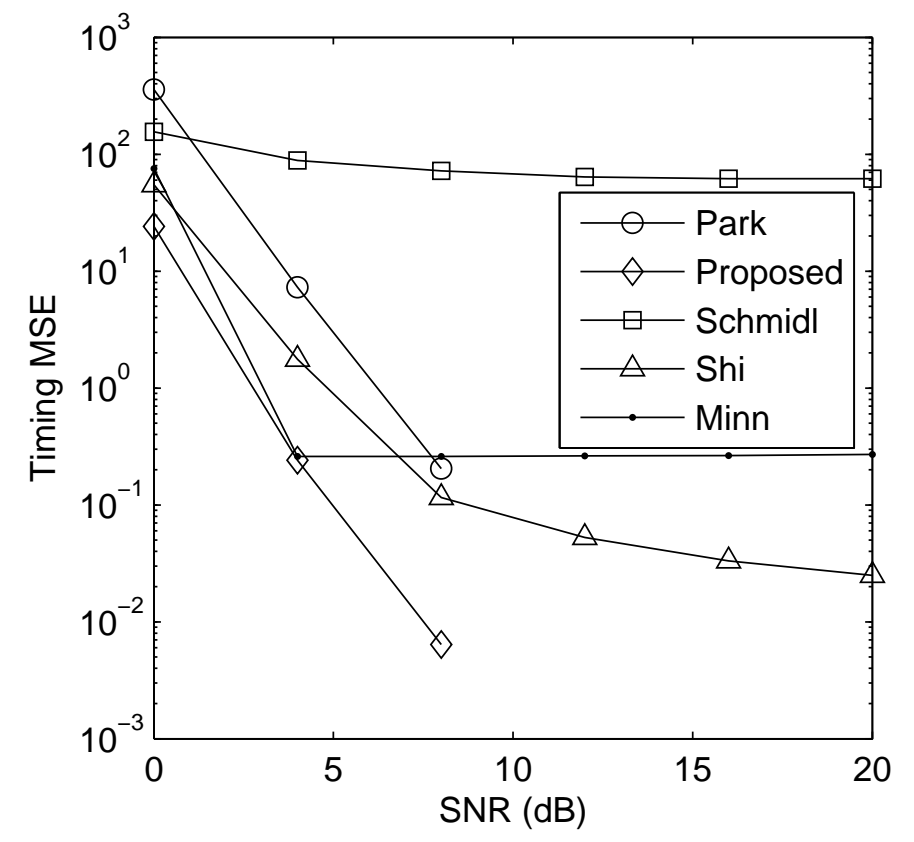


Figure 3

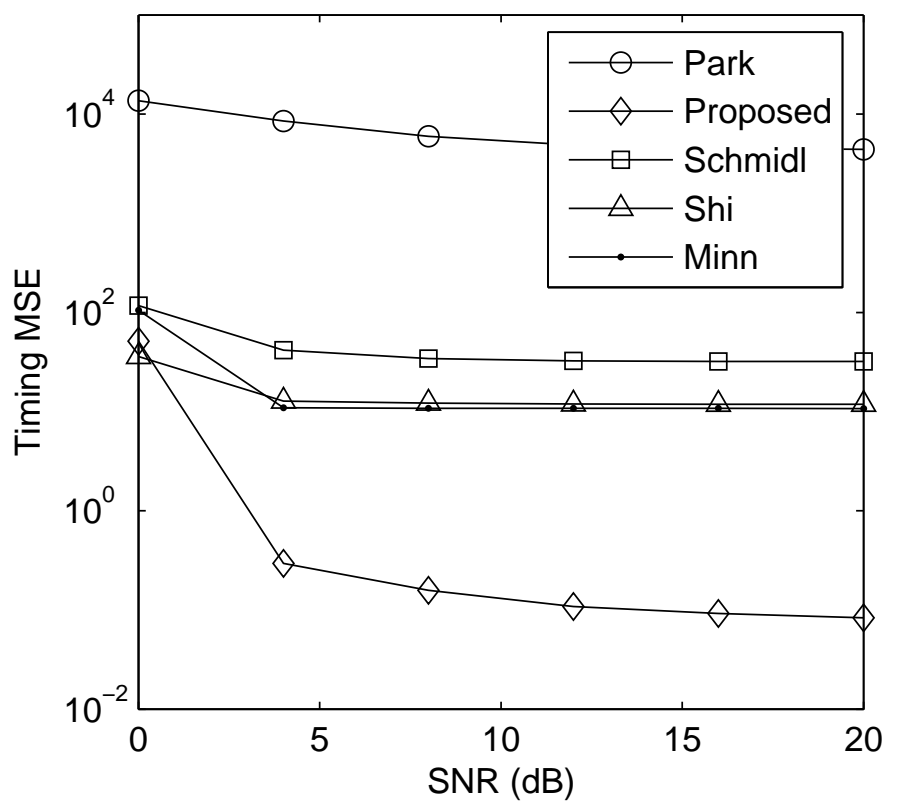

